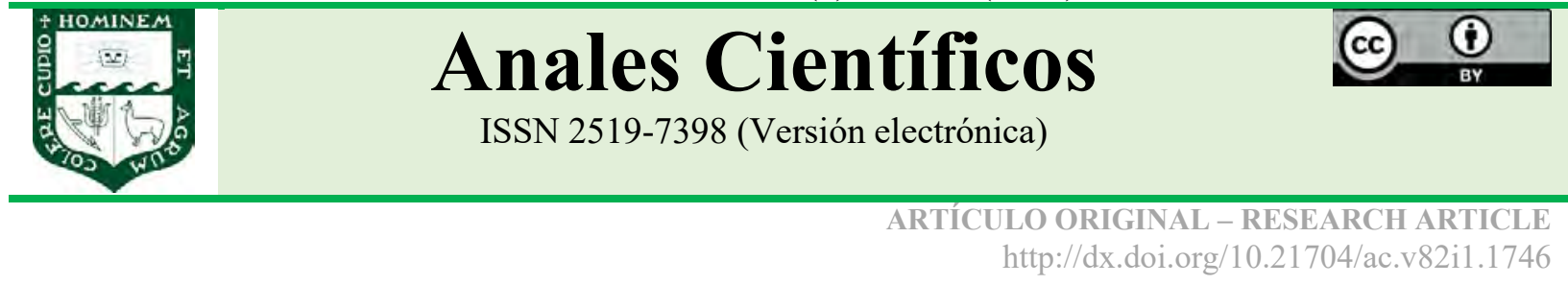

\title{
ANÁLISIS DE FRECUENCIA DE LA PRECIPITACIÓN MENSUAL EN LA REGIÓN JUNÍN, PERÚ
}

\author{
Analysis of the frequency of the monthly rainfall in the region Junin, Peru \\ Liz M. Palomino Zegarra ${ }^{1 *}$; $;$ Erick A. Rosales Asto ${ }^{10}$; Jorge L. Cárdenas Guillén ${ }^{1}(\mathbb{C}$ \\ ${ }^{1}$ Facultad de Ingeniería Agrícola, Universidad Nacional Agraria La Molina, 15024, Lima, Perú. \\ *E-mail: lizpalomino@lamolina.edu.pe
}

Recibido: 28/11/2019; Aceptado: 02/03/2021; Publicado: 31/08/2021

\begin{abstract}
This work aimed to perform the analysis of monthly precipitation frequency for the departments of Huancavelica, Junín and Pasco. The analysis of monthly precipitation frequency was carried out with rainfall information evaluated for the period from January 1965 to December 2001 of 19 stations which are distributed according to the climatic characteristics and the homogeneity of the data in the study area, which includes the departments of Huancavelica, Junín and Pasco. Among the results of the study were identified regions that represent the monthly precipitation which are based on probability distribution laws: Exponential, Generalized Pareto, Weibull, Gumbel, GEV, Normal. Log-Normal, Gamma, Pearson type III, Log Pearson and Poisson exponential. The probability distribution laws that fit the monthly precipitation in zone I are Log-Normal and Gamma and for Zone II Gamma and Log-Normal.

Keywords: AED; regionalization; frequency analysis; monthly rainfall; regional vector
\end{abstract}

\section{RESUMEN}

El presente trabajo tuvo como objetivo realizar el análisis de frecuencia de precipitación mensual en los departamentos de Huancavelica, Junín y Pasco. El análisis de frecuencia de precipitación mensual se realizó con información pluviométrica evaluadas para el período comprendido entre enero de 1965 a diciembre del 2001 de 19 estaciones el cual se encuentran distribuidos de acuerdo a las características climáticas y de homogeneidad de los datos en el área de estudio, que comprende los departamentos de Huancavelica, Junín y Pasco. Entre los resultados del estudio se identificó regiones que permite representar la precipitación mensual el cual están basados en leyes de distribución de probabilidad: Exponencial, Pareto generalizado, Weibull, Gumbel, GEV, Normal. Log-Normal, Gamma, Pearson tipo III, Log Pearson y Poisson exponencial. Las leyes de distribución de probabilidad que se ajustan a la precipitación mensual en la zona I son Log-Normal y Gamma y para la zona II Gamma y Log-Normal.

Palabras clave: AED; regionalización; análisis de frecuencia; precipitación mensual; vector regional.

Forma de citar el artículo (Formato APA):

Palomino-Zegarra, L., Rosales, E., \& Cárdenas, J. (2021) Análisis de frecuencia de la precipitación mensual en la región Junín, Perú. Anales Científicos. 82(1), 98-105. http://dx.doi.org/10.21704/ac.v82i1.1746

Autor de correspondencia (*): Liz Palomino-Zegarra. Email: lizpalomino@lamolina.edu.pe

(C) Los autores. Publicado por la Universidad Nacional Agraria La Molina.

This is an open access article under the CC BY 


\section{INTRODUCCIÓN}

El comportamiento de las variables en un sistema hidrológico se describe con la ayuda de funciones de distribución de probabilidades, particularmente en la estimación de sucesos extremos correspondientes a diferentes periodos de retorno. La magnitud del evento extremo está relacionada con su frecuencia de ocurrencia (Aparicio, 2003).

La sequía meteorológica es definida a partir de un umbral de déficit de precipitación que se alcanza durante un período de tiempo previamente determinado (Acuña-Arboleda et al., 2011), el déficit de precipitación es variable en cada región debido a las condiciones atmosféricas. Estas sequías ocasionan grandes impactos económicos, sociales y medio ambientales, sobre todo en regiones áridas y semiáridas, como es el caso de algunas partes del Perú. Es importante lograr comprender las sequías meteorológicas, para ello se debe conocer la probabilidad de ocurrencia en distintos niveles de severidad y duración (Acuña-Arboleda et al., 2011).

Fenómenos como "El Niño" provocan eventos climáticos extremos y cambiantes a nivel mundial y el Perú también es afectado por dicho fenómeno, provocando sequías donde antes existía frecuencia de lluvias y provocando lluvias donde antes no las había, generando grandes impactos en la población, siendo imprescindible realizar análisis de variabilidad de precipitación a nivel regional (León, 2014).

En la región Junín, la predictibilidad de las lluvias es desconocida, dar un pronóstico mensual de la intensidad de lluvia es muy difícil debido a las características geográficas de la zona e influenciada por fenómenos físicos que hacen que esta zona se vea expuesta a la variabilidad climática. En el presente trabajo se analizarán las precipitaciones mensuales en la Región Junín para establecer regiones con las leyes de distribución de probabilidad.

\section{MATERIALES Y MÉTODOS}

\section{Área de estudio y datos de precipitación}

El área de estudio está localizada en la cuenca del río Mantaro, integrando a su vez el sistema hidrográfico del Amazonas, políticamente esta cuenca abarca los departamentos de Ayacucho, Huancavelica, Junín y
Pasco entre los $10^{\circ} 30^{\prime}-13^{\circ} 30^{\prime}$ de latitud sur y los $74^{\circ} 00^{\prime}-76^{\circ} 30^{\prime}$ de longitud oeste (Figura 1).

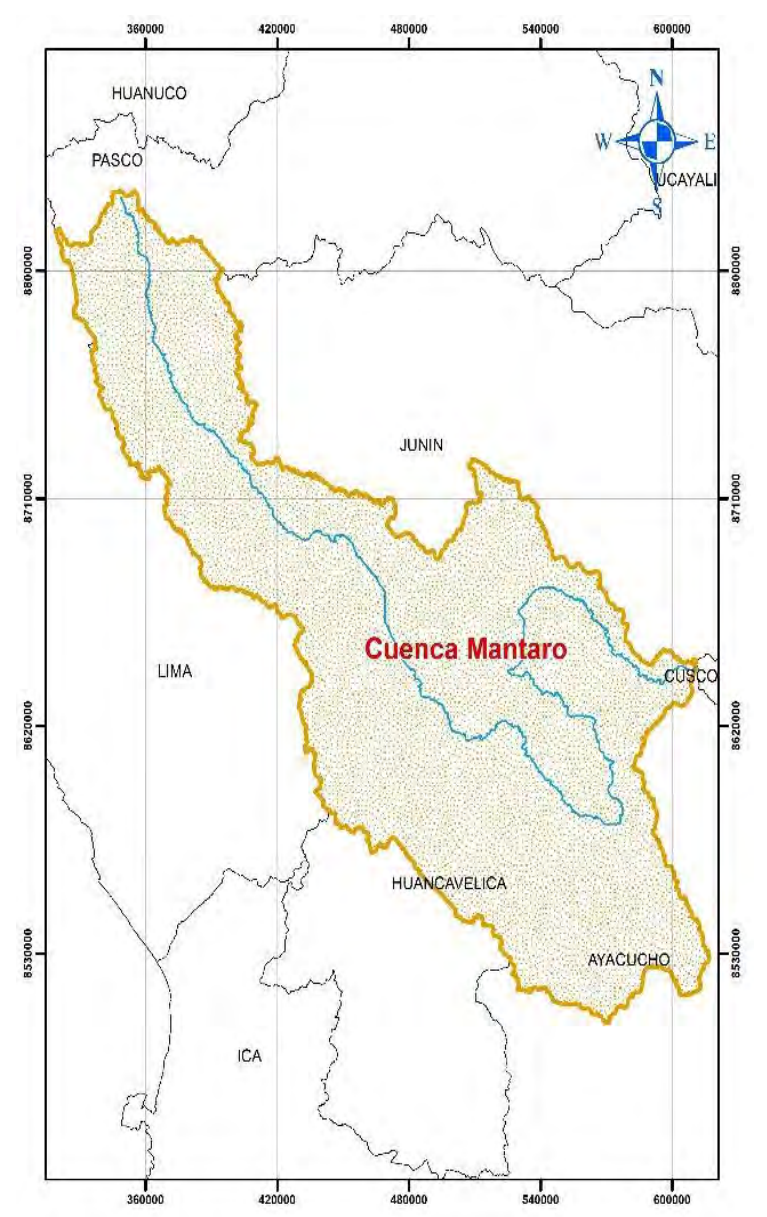

Figura 1. Área de estudio del presente trabajo.

El análisis se realizó mes a mes para cada estación en cada zona para el cual se utilizó el software HYFRAN versión 1.1 basado en leyes de probabilidad: Exponencial, Pareto generalizado, Weibull, Gumbel, GEV, Normal. Log-Normal, Gamma, Pearson tipo III, Log Pearson y Poisson exponencial.

Los datos de precipitación diaria fueron descargados del Servicio Nacional de Meteorología e Hidrología del Perú, de donde se obtuvo datos de 60 estaciones ubicadas en el área de estudio y estaciones correspondientes a cuencas aledañas del cual 27 pertenecen a SENAMHI y 33 a Electroandes S.A (Tabla 1). 
Tabla 1. Información de las estaciones pluviométricas utilizadas.

\begin{tabular}{|c|c|c|c|c|}
\hline Estación & Cuenca & Latitud & Longitud & Altitud \\
\hline Santa Ana & Mantaro & $-12,0042$ & $-75,2208$ & 3295 \\
\hline Viques & Mantaro & $-12,1633$ & $-75,2353$ & 3186 \\
\hline Ingenio & Mantaro & $-11,8808$ & $-75,2878$ & 3390 \\
\hline Ricran & Perene & $-11,5394$ & $-75,2878$ & 3820 \\
\hline Carhuacayan & Mantaro & $-11,2000$ & $-76,2833$ & 4150 \\
\hline Huayao & Mantaro & $-12,0336$ & $-75,2669$ & 3225 \\
\hline Yantac & Mantaro & $-11,3333$ & $-76,4000$ & 4600 \\
\hline Jauja & Mantaro & $-11,7866$ & $-75,4869$ & 3378 \\
\hline Pariacancha & Chillón & $-11,3932$ & $-76,5167$ & 3800 \\
\hline Rio Blanco & Rímac & $-11,7345$ & $-76,2589$ & 3350 \\
\hline Vilca & Cañete & $-12,1146$ & $-75,8262$ & 3864 \\
\hline Yauricocha & Cañete & $-12,3331$ & $-75,7226$ & 4675 \\
\hline Huaros & Chillón & $-11,4000$ & $-76,5667$ & 3585 \\
\hline Pirca & Huaral & $-11,2328$ & $-76,6551$ & 3320 \\
\hline Santa cruz & Huaral & $-11,1901$ & $-76,6349$ & 3576 \\
\hline Parquin & Huaura & $-10,9667$ & $-76,7167$ & 3590 \\
\hline Picoy & Huaura & $-10,9167$ & $-76,7333$ & 2990 \\
\hline Oyon & Huaura & $-10,6677$ & $-76,7687$ & 3650 \\
\hline Tanta & Cañete & $-12,1167$ & $-76,0167$ & 4323 \\
\hline Carania & Cañete & $-12,3444$ & $-75,8721$ & 3875 \\
\hline San Rafael & Huallaga & $-10,3292$ & $-76,1766$ & 2722 \\
\hline Salcabamba & Mantaro & $-12,1994$ & $-74,7858$ & 3280 \\
\hline Acostambo & Mantaro & $-12,3661$ & $-75,0564$ & 3675 \\
\hline Huancalpi & Mantaro & $-12,5394$ & $-75,2367$ & 3450 \\
\hline Acobamba & Mantaro & $-12,8531$ & $-74,5603$ & 3236 \\
\hline Lircay & Mantaro & $-12,9819$ & $-12,9819$ & 3360 \\
\hline Tunel cero & Pampas & $-13,2593$ & $-75,0860$ & 4512 \\
\hline Casapalca & Yauli & $-11,5833$ & $-76,0597$ & 4225 \\
\hline Curipata (cut off) & Yauli & $-11,6014$ & $-75,9708$ & 3900 \\
\hline Huallacocha & Yauli & $-11,7708$ & $-76,0931$ & 4371 \\
\hline Huascacocha & Yauli & $-11,5889$ & $-76,0833$ & 4370 \\
\hline Morococha & Yauli & $-11,6014$ & $-76,1347$ & 4505 \\
\hline Pachachaca & Yauli & $-11,6181$ & $-76,0333$ & 3979 \\
\hline Pomacocha & Yauli & $-11,7111$ & $-76,1139$ & 4305 \\
\hline Pucara & Yauli & $-11,5833$ & $-76,0597$ & 4225 \\
\hline Atocsaico & Mantaro & $-11,2986$ & $-76,0778$ & 4200 \\
\hline Carhuamayo & Mantaro & $-10,9236$ & $-76,0569$ & 4125 \\
\hline Casapato & Mantaro & $-10,9806$ & $-76,0389$ & 4100 \\
\hline Corpacancha & Mantaro & $-11,3653$ & $-76,2153$ & 4150 \\
\hline Hda. Huanca & Mantaro & $-10,6792$ & $-76,0833$ & 4150 \\
\hline Malpaso & Mantaro & $-11,4097$ & $-76,0417$ & 3795 \\
\hline Paragsha & Mantaro & $-10,6736$ & $-76,2569$ & 4310 \\
\hline San Blas & Mantaro & $-11,1028$ & $-76,1819$ & 4300 \\
\hline Shelby & Mantaro & $-10,8097$ & $-76,2250$ & 4120 \\
\hline Upamayo & Mantaro & $-10,9250$ & $-76,2778$ & 4093 \\
\hline Altos Machay & Paucartambo & $-10,5514$ & $-75,8833$ & 4140 \\
\hline Chalhuacocha & Huachon & $-10,5889$ & $-75,8597$ & 4050 \\
\hline Huachon & Paucartambo & $-10,6306$ & $-75,9472$ & 3400 \\
\hline Huallamayo & Paucartambo & $-10,7597$ & $-75,7153$ & 2440 \\
\hline Huangush Alto & Paucartambo & $-10,5722$ & $-75,8236$ & 3885 \\
\hline Huangush Bajo & Paucartambo & $-10,5833$ & $-75,8069$ & 3600 \\
\hline Jaico & Paucartambo & $-10,5556$ & $-75,9125$ & 4230 \\
\hline La victoria Ii & Paucartambo & $-10,8583$ & $-75,9222$ & 4000 \\
\hline Lechecocha & Paucartambo & $-10,5264$ & $-75,9000$ & 4220 \\
\hline Luxopata & Paucartambo & $-10,8361$ & $-75,8583$ & 4000 \\
\hline Machavado & Paucartambo & $-10,5806$ & $-75,8972$ & 3850 \\
\hline Manto & Paucartambo & $-10,7403$ & $-75,5847$ & 1870 \\
\hline Pacchapata & Paucartambo & $-10,5444$ & $-75,8958$ & 4360 \\
\hline Paucartambo & Paucartambo & $-10,7667$ & $-75,8111$ & 3000 \\
\hline Yuncan & Paucartambo & $-10,7167$ & $-75,6500$ & 1870 \\
\hline
\end{tabular}

En el presente trabajo se ha considerado la longitud de las series de los datos de precipitación para el período de análisis comprendido entre los años 1965 a 2001.

\section{RESULTADOS Y DISCUSIÓN}

\section{Análisis exploratorio de datos}

Para la detección y la comprobación de la calidad de los datos se realizó el análisis exploratorio de datos con la finalidad de conseguir un entendimiento básico de los datos y verificar la existencia de datos atípicos para comprobar las propiedades de homogeneidad en la información original (León, 2014).

El análisis se realizó sobre datos de precipitación mensual en el que se depuró los datos atípicos. En primera instancia del análisis exploratorio de datos se realizó el análisis de los datos atípicos (Figura 2).

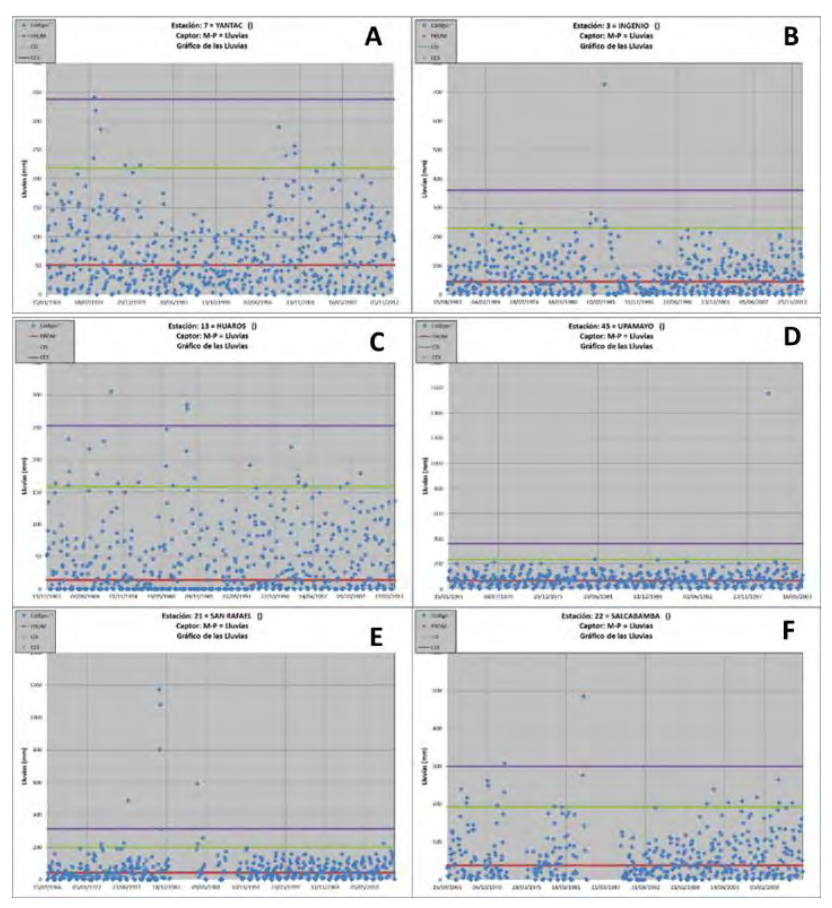

Figura 2. Identificación de valores atípicos. Gráfico de lluvias. A) Estación 7=Yantac; B) Estación 3=Ingenio; C) Estación=Huaros; D) Estación=Upamayo; E) Estación 21=San Rafael; F) Estación=Salcabamba.

\section{Zonas climatológicamente homogéneas.}

Para un mejor análisis de los datos para el área de estudio, las estaciones se han dividido en regiones 
climatológicamente homogéneas, es decir en regiones cuyos climas están influenciados con características similares. El área de estudio presenta clima semiseco frío correspondiendo a los valles interandinos bajos e intermedios, situados entre los 1000 y $3000 \mathrm{msnm}$, clima lluvioso semifrigido (de tundra) que se extiende entre 3500 y $6000 \mathrm{msnm}$ y un clima lluvioso frío que se extiende entre los 3000 y $4000 \mathrm{msnm}$.

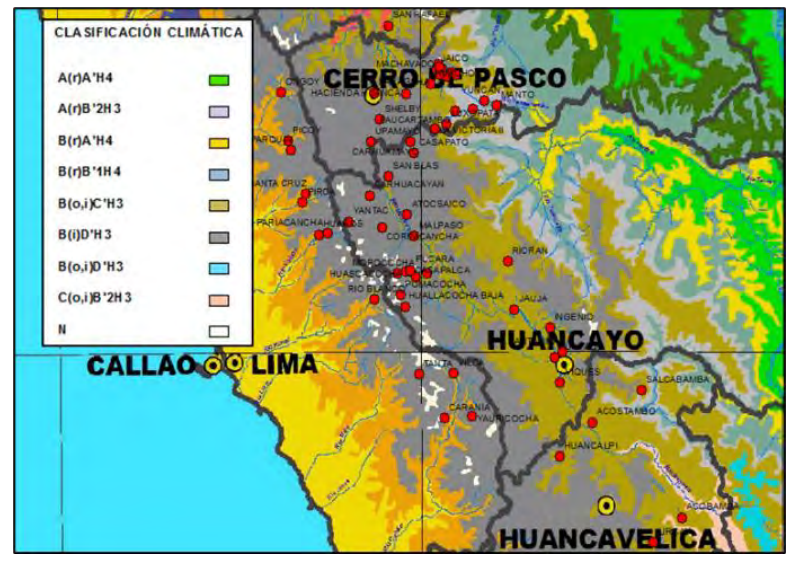

Figura 3. Ubicación geográfica de las estaciones pluviométricas en el mapa climático. A(r) A'H4: Clima cálido, muy lluvioso-abundante precipitación, $\mathrm{A}(\mathrm{r})$ B'2H3: Clima templado, muy lluvioso- abundante precipitación, B(r) A’H4: Clima cálido, muy lluviosoabundante precipitación, B(r) B'1H4: Clima semicálido, muy lluvioso-abundante precipitación, $\mathrm{B}(\mathrm{o}, \mathrm{i}) \mathrm{C}^{\prime} \mathrm{H} 3$ : Clima frio, lluvioso-deficiencia de lluvia en otoño e invierno. B(i) D'H3: Clima semifrígido, lluvioso-deficiencia de lluvia en invierno, B(o,i) D'H3: Clima semifrígido, lluvioso-deficiencia de lluvia en otoño e invierno, C(o,i) B'2H3: Clima templado, semiseco-deficiencia de lluvia en otoño e invierno.

FUENTE: Modificado de SENAMHI.

Tabla 2. Agrupación climática y orográfica de estaciones.

\begin{tabular}{ll}
\hline Zona I & Zona II \\
B(i)D'H3 & B(o,i)C'H3 \\
\hline Jauja & Altos Machay \\
Acostambo & Chalhuacocha \\
Lircay & Huangush Alto \\
Curipata (Cut Off) & Huangush Bajo \\
Huallacocha Baja & Jaico \\
Huascacocha & Lechecocha \\
Morococha & \\
Pachachaca & \\
Pomacocha & \\
Casapato & \\
Corpacancha & \\
Malpaso & \\
Upamayo & \\
\hline
\end{tabular}

\section{Complementación de datos y regionalización}

El método del vector regional se trata de un método de cálculo orientado a dos tareas definidas: La crítica de datos y la homogenización. La idea básica del método es la siguiente: en lugar de comparar pares de estaciones por correlación o doble masa, como se realiza en los métodos clásicos, se elabora una estación ficticia que sea una "especie de promedio" de todas las estaciones de la zona, con la cual se comparan cada una de las estaciones. En lugar de emplear un promedio simple, utiliza el concepto de la precipitación media extendida, evitando los problemas de peso de las estaciones y la falta de datos (Espinoza, 2005).

Para el presente trabajo empleamos el software Hydraccess versión 4.6 el cual tiene incluido un módulo para el cálculo del vector regional por el método de Y. Brunet Moret. Asimismo, el software nos permitió calcular el vector regional a paso de tiempo mensual y anual (análisis y corrección). Igualmente nos permitió detectar, corregir o eliminar los errores sistemáticos de series cronológicas estudiadas (fase de homogenización) luego de buscar las zonas climáticas homogéneas (fase de regionalización) para las cuales se generó una serie cronológica representativa de índices mensuales y anuales.

Para el cálculo del vector regional se consideró tener como mínimo 3 estaciones por año, y 3 años como mínimo por estación. Se consideró una zona homogénea si los coeficientes de correlación anual entre el vector y la estación son superiores o iguales a 0.7 (Ministerio de Agricultura, 2007). Cada una de las zonas y el respectivo análisis de los registros pluviométricos correspondientes se muestran a continuación.

Los resultados del vector regional de la Zona I y la Zona II se muestran en la Tabla 3 y Tabla 4 respectivamente donde antes de generar el vector se realizó el análisis exploratorio de datos agrupando de forma visual el comportamiento multianual y el comportamiento mensual. Los resultados estadísticos respecto al vector regional como el coeficiente de correlación es mayor a 0,7 y la desviación estándar de desvío (D.E. Desvíos) es menor a 0,2 , presentándose en la Zona I y en la Zona II. La estimación de datos faltantes mensuales se realizó por medio del vector regional. 
Tabla 3. Resultados del vector regional de la Zona I.

\begin{tabular}{lcccc}
\hline \multicolumn{1}{c}{ Id Estación } & $\begin{array}{c}\# \\
\text { Años }\end{array}$ & $\begin{array}{c}\text { D.E. } \\
\text { Obs. }\end{array}$ & $\begin{array}{c}\text { D.E. } \\
\text { Desvíos }\end{array}$ & $\begin{array}{c}\text { Correl. } \\
\text { /Vector }\end{array}$ \\
\hline Altos Machay & 36 & 190,4 & 0,075 & 0,809 \\
Chalhuacocha & 27 & 156,2 & 0,031 & 0,946 \\
Huangush Alto & 37 & 180,3 & 0,072 & 0,724 \\
Huangush Bajo & 37 & 175,8 & 0,067 & 0,746 \\
Jaico & 37 & 182,3 & 0,064 & 0,846 \\
Lechecocha & 33 & 141,6 & 0,062 & 0,762 \\
\hline
\end{tabular}

\section{Análisis de estacionariedad}

El criterio de estacionariedad implica que los datos deben estar libres de tendencia durante el periodo de observación. Después del análisis exploratorio a cada serie de precipitación, se determinaron tendencias a escalas anual, Para ello, se utilizó el test no paramétrico Estacional de Mann-Kendall, Distribución Free CUSUN y Rank - Sum el cual indica de no hay tendencia.

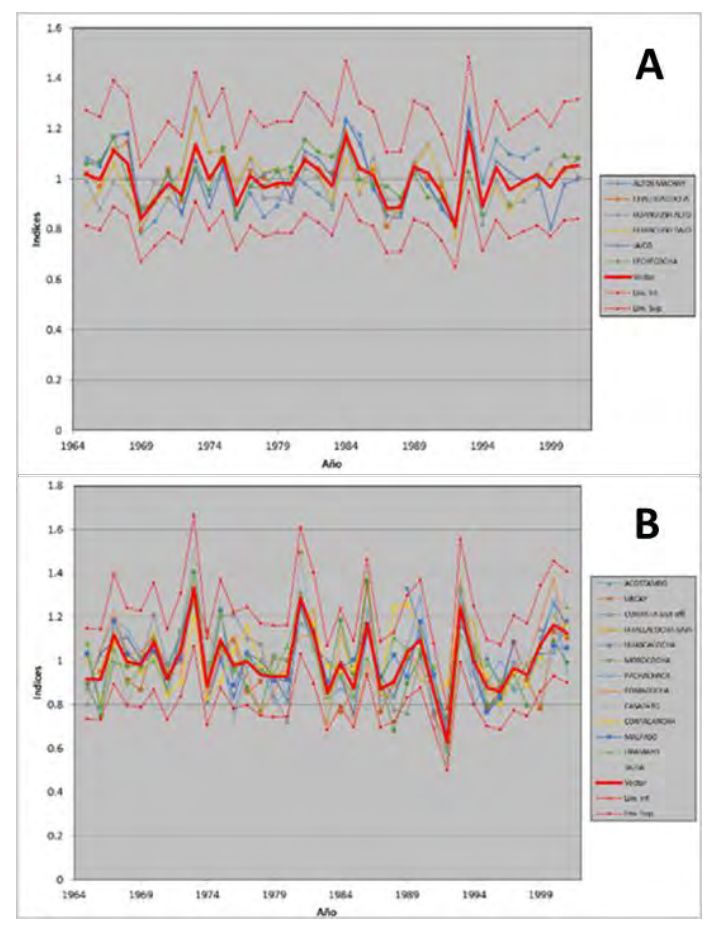

Figura 4. Vector regional Zona I (A) y II (B). Índices anuales del vector y de las estaciones (Brunet Moret).

\section{Análisis de distribución de probabilidad}

Se realizó el análisis de distribución de probabilidad mes a mes para cada estación en cada zona para el cual empleamos el software HYFRAN versión 1.1 basado en leyes de probabilidad: Exponencial, Pareto generalizado, Weibull, Gumbel, GEV, Normal. LogNormal, Gamma, Pearson tipo III, Log Pearson tipo II, Lognormal (3 parámetros).

HyFRAN (análisis de frecuencia hidrológica), desarrollado por el Instituto Nacional de Investigación Científica - Agua, tierra y medio ambiente (INRS-ETE) de la Université du Québec. Inicialmente diseñado para análisis de frecuencia de eventos extremos, HyFRAN puede usarse en cualquier estudio que requiera ajustando una distribución estadística a una serie de datos independientes e idénticamente distribuidos (Merzougui y Slimani, 2012).

Tabla 4. Resultados del vector regional de la Zona II (*)

\begin{tabular}{lcccc}
\hline \multicolumn{1}{c}{ Id Estación } & $\begin{array}{c}\# \\
\text { Años }\end{array}$ & $\begin{array}{c}\text { D.E. } \\
\text { Obs. }\end{array}$ & $\begin{array}{c}\text { D.E. } \\
\text { Desvíos }\end{array}$ & $\begin{array}{c}\text { Correl. } \\
\text { /Vector }\end{array}$ \\
\hline Acostambo & 12 & 139,2 & 0,111 & 0,828 \\
Lircay & 30 & 161,1 & 0,115 & 0,807 \\
Curipata (Cut off) & 34 & 121,3 & 0,101 & 0,832 \\
Huallacocha Baja & 36 & 129,3 & 0,071 & 0,89 \\
Huascacocha & 36 & 126 & 0,075 & 0,878 \\
Morococha & 36 & 144,8 & 0,1 & 0,809 \\
Pachachaca & 37 & 127,9 & 0,097 & 0,842 \\
Pomacocha & 37 & 123,7 & 0,094 & 0,825 \\
Casapato & 37 & 131 & 0,14 & 0,714 \\
Corpacancha & 36 & 119,3 & 0,108 & 0,69 \\
Malpaso & 34 & 115,8 & 0,07 & 0,878 \\
Upamayo & 36 & 125 & 0,085 & 0,816 \\
Jauja & 10 & 149 & 0,077 & 0,968 \\
\hline
\end{tabular}

(*) Estimación de datos faltantes mensuales se realizó por medio del vector regional.

Una vez que una región homogénea ha satisfecho la condición de homogeneidad es posible, entonces, determinar la función de distribución de probabilidad de mejor ajuste (Acuña-Arboleda et al., 2011).

Los resultados del vector regional de la Zona I y la Zona II se muestran en la Tabla 3 y Tabla 4 respectivamente donde antes de generar el vector se realizó el análisis exploratorio de datos agrupando de forma visual el comportamiento multianual y el comportamiento mensual. Los resultados estadísticos respecto al vector 
regional como el coeficiente de correlación es mayor a 0,7 y la desviación estándar de desvío (D.E. Desvíos) es menor a 0,2, presentándose en la Zona I y en la Zona II. La estimación de datos faltantes mensuales se realizó por medio del vector regional.

En la Figura 6 se muestra los pesos de las leyes que mejor se ajustan a la serie de precipitación mensual adoptadas en la Zona I, para distintos meses, excepto los meses secos (abril, mayo, junio, julio, agosto y setiembre). La limitación de estas tres leyes es que no comparten un ajuste entre sí en el cual no se compensan en ningún mes del año para la Zona I, y solo comparten un ajuste casi del 45\% entre la distribución Lognormal y la distribución Gama para los meses de octubre, noviembre y enero para la Zona II

En la Figura 7 se muestra las leyes de distribución de probabilidad de la precipitación mensual para la Zona I y II de la zona de la Cuenca del rio Mantaro.
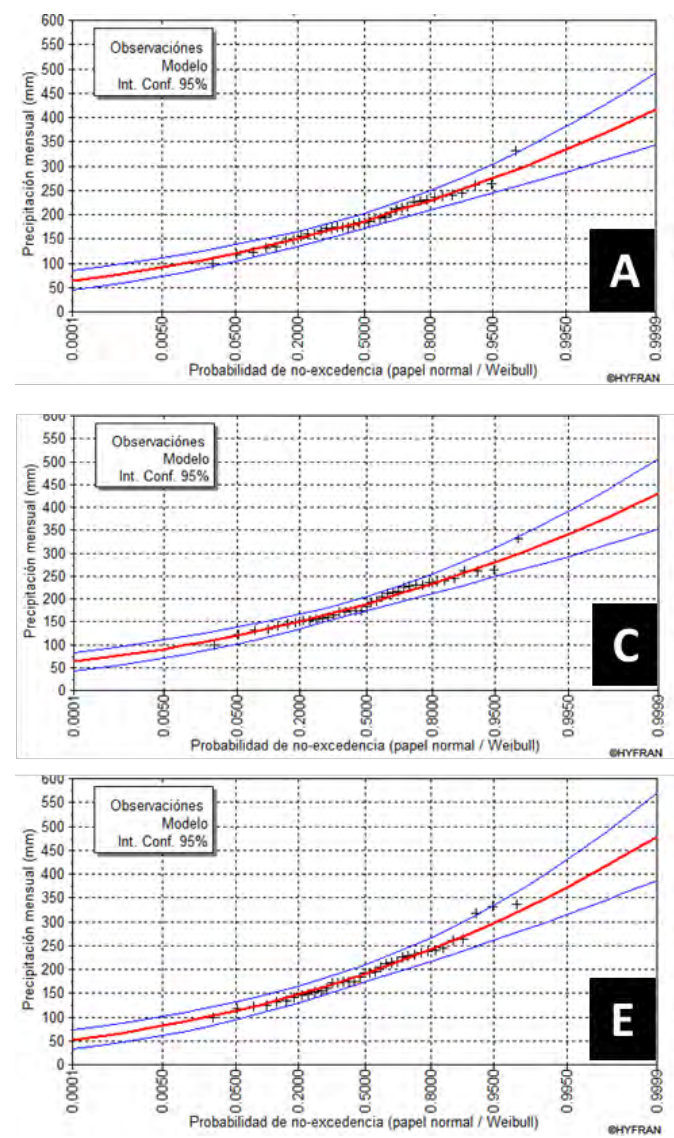

La Zona I enmarcada en el círculo de color azul muestra las estaciones donde para los meses de octubre, noviembre, enero, febrero y marzo se ajustan mejor la distribución Lognormal. Mientras que para el mes de diciembre se ajusta mejor la distribución Gamma. La Zona II enmarcada con círculo rojo muestra las estaciones al Noroeste donde la distribución de mayor ajuste para los meses de octubre, noviembre, diciembre y enero es la distribución Gamma y la distribución lognormal para los meses de febrero y marzo.

Las estaciones al Sureste donde la distribución de mayor ajuste para los meses de octubre, noviembre, diciembre y enero se ajusta mejor la distribución Lognormal y la distribución Gamma para los meses de febrero y marzo. Tras el ajuste de la serie de precipitaciones medias mensuales, para los diferentes meses y las 27 estaciones seleccionadas se muestran los criterios de comparación de las distribuciones a través de los Criterios de Información Bayesianos (BIC) y Akaike (AIC).
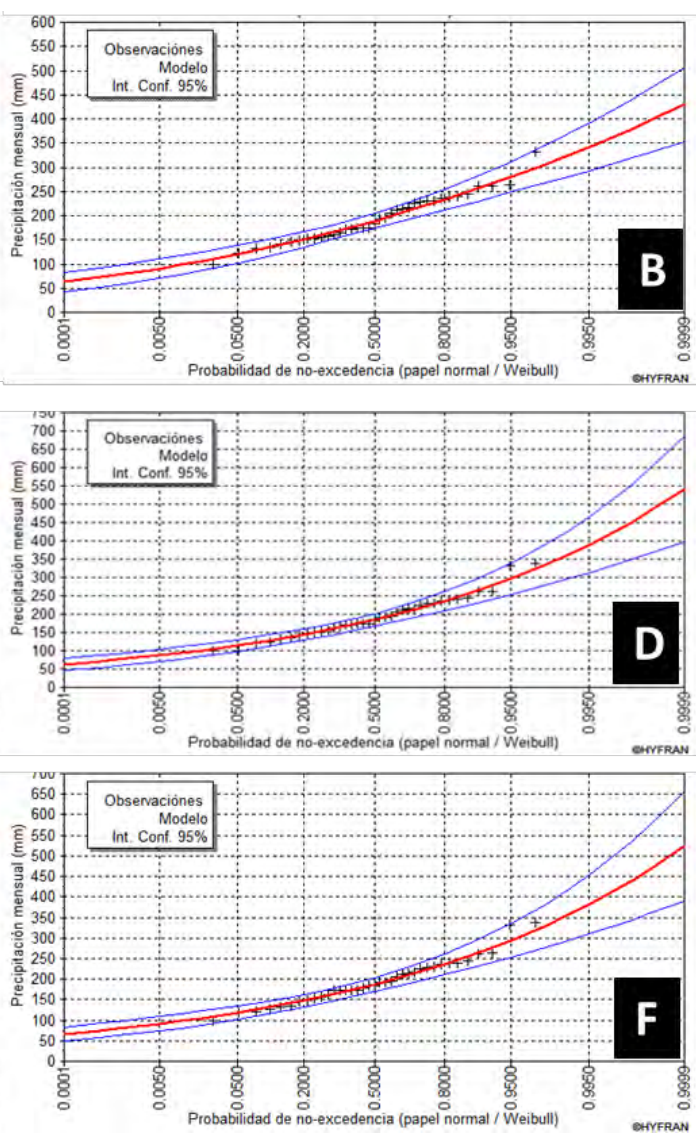

Figura 5. Leyes de distribución de probabilidad - Estación Lechecocha. Gamma (Método de momentos). Estación Lechecocha: A) Octubre; B) Noviembre; C) Diciembre; D) Enero; E) Febrero; F) Marzo. 

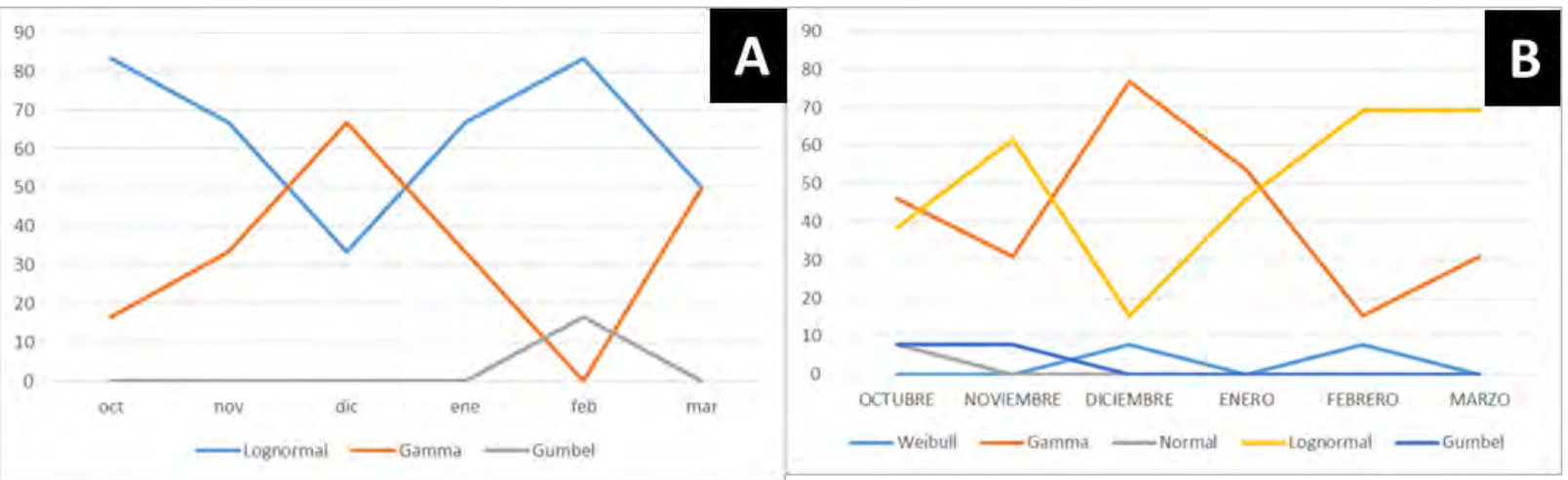

Figura 6. Distribución mensual de las dos mejores leyes. A) Zona I y B) Zona II.

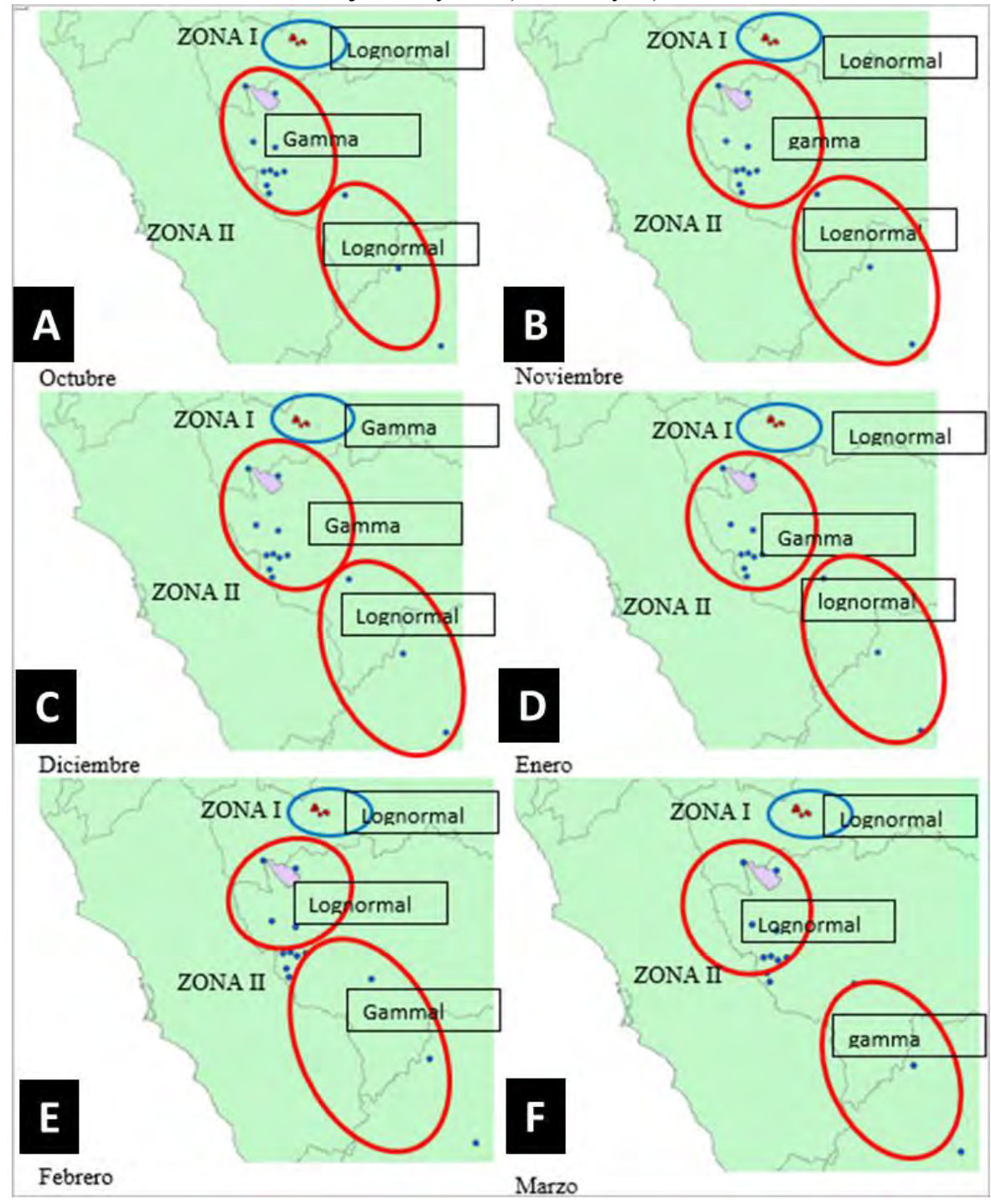

Figura 7. Distribución espacial de las Leyes de distribución de probabilidad de la precipitación mensual para la Zona I y II. A) Octubre; B) Noviembre; C) Diciembre; D) Enero; E) Febrero; F) Marzo. 


\section{CONCLUSIONES}

Se han identificado dos regiones hidrológicamente homogéneas que corresponden a clima lluvioso semifrigido (de tundra) que se extiende entre $3500 \mathrm{y}$ $6000 \mathrm{msnm}$ y un clima lluvioso frío que se extiende entre los 3 mil y 4 mil msnm. La completación de datos se realizó con el vector regional considerando las Zona I y II homogéneas y que la desviación estándar de desvíos fue menor a 0,2 y el coeficiente de correlación fue mayor a 0,7. Se utilizó el test no paramétrico Estacional de Mann-Kendall, Distribución Free CUSUN y Rank - Sum el cual indica que no hay tendencia para la precipitación total anual para el periodo de 1965 al 2001.

Las leyes de distribución de probabilidad analizadas para cada estación fueron Exponencial, Pareto generalizado, Weibull, Gumbel, GEV, Normal. LogNormal, Gamma, Pearson tipo III dicho análisis se realizó para los meses de octubre, noviembre, diciembre, enero, febrero y marzo, cuyo criterio de comparaciones permitió encontrar la mejor distribución en la zona I (Log-Normal y Gamma) y para la zona II (Gamma y Log-Normal) basados en los parámetros de AIC (Criterio de Información de Akaike) y BIC (Criterio de Información Bayesiano).

\section{Conflictos de intereses}

Los autores firmantes del presente trabajo de investigación declaran no tener ningún potencial conflicto de interés personal o económico con otras personas $\mathrm{u}$ organizaciones que puedan influir indebidamente con el presente manuscrito.

\section{Contribuciones de los autores}

Preparación y ejecución: LP, ER, JC; Desarrollo de la metodología: LP, ER, JC; Concepción y diseño: LP, ER, JC; Edición del artículo: LP, ER, JC; Supervisión del estudio: LP, ER, JC;

\section{LITERATURA CITADA}

- Acuña, J., Felipe, O., Ordoñez, J., \& Arboleda, F. (2011). Análisis regional de frecuencia de precipitación anual para la determinación de mapas de sequía. Revista Peruana Geo-Atmosfera RPGA (3), 104-115.

- Aparicio, J. (2003). Fundamentos de Hidrología de superficie, editorial Limusa México. 239-241.

- Espinoza, J. (2005) Utilización del método del Vector Regional con HYDRACCESS. SENAMHI Perú. Septiembre. Colaboración de Philippe Vauchel (IRD-Peru) y Josyane Ronchail (UP7 LOCEAN, Paris). 9 p. Disponible en: http://sites.google.com/site/jhancarloespinoza

- Leon, K. (2014). Análisis espacio-temporal de las precipitaciones y caudales durante los eventos el niño (1982-83 y 1997-98) en la costa norte peruana UNALM. 26-28p., 55-61 p.

- Merzougui, A., \& Slimani, M. (2012). Régionalisation des lois de distribution des pluies mensuelles en Tunisie. Hydrological Sciences Journal. 57(4), 668-685

- Ministerio de Agricultura. Instituto Nacional De Recursos Naturales, Intendencia De Recursos Hídricos 2007. "Evaluación De Los Recursos Hídricos De La Cuenca Del Río Ocoña. Volumen I. 83-91 p. Disponible en: https://hdl.handle.net/20.500.12543/3886.

- Senamhi [Servicio Nacional de Meteorología e Hidrología del Perú]. 2018. Mapa Climático Nacional. Disponible en https://web2.senamhi.gob.pe/?p=mapa-climaticodel-peru.

- Senamhi [Servicio Nacional de Meteorología e Hidrología del Perú]. 2018. Mapa de Clasificación Climática del Perú. Disponible en https://idesep.senamhi.gob.pe/geonetwork/srv/spa/ catalog.search\#/metadata/9f18b911-64af-4e6bbbef-272bb20195e4. 